\title{
Korean VLBI Network receiver optics for simultaneous multi-frequency observation
}

\author{
Seog-Tae Han ${ }^{1}$ \\ Korea Astronomy and Space Science Institute \\ 776 Daedeokdae-ro, Yuseong-gu, Daejeon, Republic of Korea \\ E-mail: sthan@kasi.re.kr
}

Jung-Won Lee, Jiman Kang, Choong-Sik Oh, Do-Young Byun, Do-Heung Je, Moon-Hee Chung, Soo-Yeon Kim, Seog-Oh Wi, Minkyu Song and Yong-Woo Kang

Korea Astronomy and Space Science Institute

776 Daedeokdae-ro, Yuseong-gu, Daejeon, Republic of Korea

\begin{abstract}
We have developed a new millimeter wave receiver system with input optics that enables simultaneous observations in four bands at 22, 43, 86 and $129 \mathrm{GHz}$. The functional goal of the optics is to facilitate calibration of tropospheric phase fluctuations in millimeter-wave VLBI observations. To make the beams of the four bands point the same position in the sky, it is crucial that errors in the alignment of these beams remain small. On-site test observations showed that the beam centers for the four bands, with reference to the $86 \mathrm{GHz}$ beam center, are aligned within 2 arcseconds over most of the elevation range of the Korean VLBI Network 21-m telescopes. Measured telescope aperture efficiencies including multiband receiver optics are $65 \%$ at $22 \mathrm{GHz}, 62 \%$ at $43 \mathrm{GHz}, 57 \%$ at $86 \mathrm{GHz}$ and $38 \%$ at $129 \mathrm{GHz}$.
\end{abstract}

11th European VLBI Network Symposium \& Users Meeting

October 9-12, 2012

Bordeaux, France

\footnotetext{
1 Speaker.
} 


\section{Introduction}

Even though various VLBI observations at millimeter and submillimeter wavelengths have been successfully performed, tropospheric phase fluctuations often reduce sensitivity and limit imaging capability. In order to reduce degradation caused by the tropospheric phase fluctuations, both water vapor radiometry and phase referencing techniques have been developed and applied to millimeter and submillimeter VLBI observations. Phase referencing techniques for millimeter-wave VLBI observations can be categorized into fast antenna or frequency switching $[1,2,3]$, paired/clustered antennas $[4,5,6,7]$, dual-beam antennas $[8,9]$. Though these phase referencing techniques are useful and reliable for phase compensation of the tropospheric phase fluctuations, their application to millimeter wave VLBI observations have been limited, due to the requirement on the separation angle between target and nearby phase calibration reference sources to achieve low residual phase errors $[10,4]$. If we could simultaneously observe a source at different observing frequencies, the residual phase errors would be eliminated because tropospheric phase variations arising from the finite switching cycle time or from the actual separation angle between target and reference source would not be present.

In order to solve the problem mentioned above for the phase referencing, the Korean VLBI Network (KVN) [11,12] employs a unique multiband receiver system that can perform simultaneous observations in four bands at 22, 43, 86 and $129 \mathrm{GHz}$. In this paper, we present details on the quasioptical circuits of the multiband receiver system and on-site test results.

\section{Receiver optics}

The complete receiver optics and receivers installed at the receiver cabins of KVN telescopes are shown in Fig. 1. The beam from the telescope subreflector is first reflected and fed to the subsequent optics by a flat $45^{\circ}$ mirror mounted on top of the receiver plate. There are three quasioptical low pass filters (LPFs). LPF1 reflects the beams of the $86 \mathrm{GHz}$ and $129 \mathrm{GHz}$ bands and transmits those of the $22 \mathrm{GHz}$ and $43 \mathrm{GHz}$ bands. Similarly, LPF2 passes the $22 \mathrm{GHz}$ beam and reflects the beam of the $43 \mathrm{GHz}$ band. LPF3 allows the $86 \mathrm{GHz}$ beam to pass while reflecting the $129 \mathrm{GHz}$ beam. Beams are formed by the receiver optics in order to have $-17 \mathrm{~dB}$ relative power level at the edge of the subreflector using focusing mirrors designed with fundamental mode Gaussian beam approximation. Each receiver is equipped with a circular polarizer following a corrugated feed horn. Details of the optics were published in [11]. 


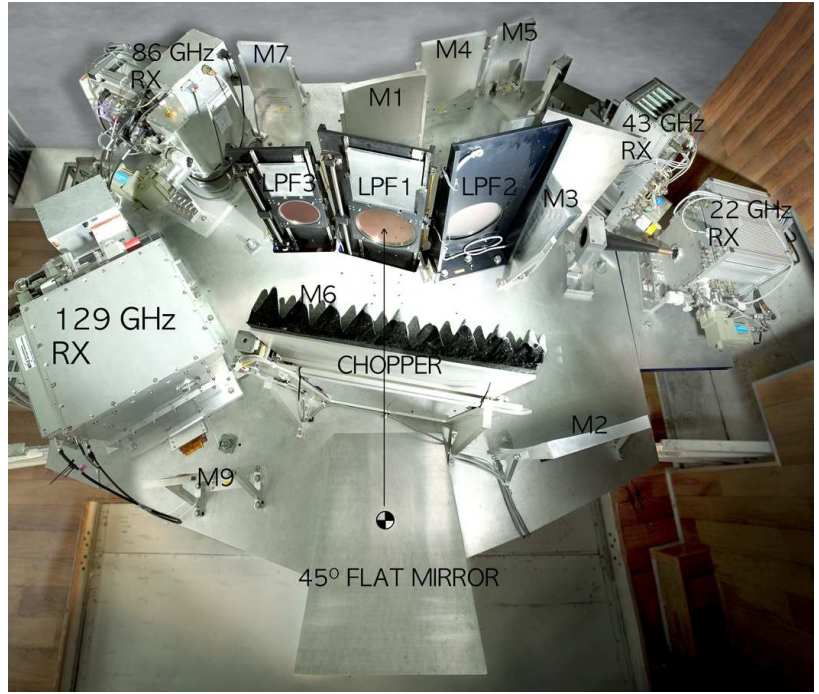

Figure 1. View of the complete quasioptical feed system installed at the receiver cabin of KVN Yonsei telescope. Each LPF is mounted onto a "mode selector", which has a flat mirror and an aperture which functions as a filter substitute. A large chopper after the flat $45^{\circ}$ mirror inserts ambient temperature into the beam path from the secondary mirror in order to calibrate the radiometers.

\section{Test results}

\subsection{Beam alignment}

To align the beams, we made successive adjustments in the laboratory while the final adjustment was done on the telescope during observations of planets or strong maser sources. The primary requirement for the alignment is that each beam be aligned to within $10 \%$ of its $3 \mathrm{~dB}$ beam width (HPBW) on the sky relative to a reference beam direction. The second requirement is to synthesize feed beams conforming to the required edge taper at the subreflector.

The measured beam patterns at the four frequencies showed circular-shaped beam patterns. This means that the quasioptical components producing the beams at the four bands are correctly aligned. The lateral and angular offsets of the beams after laboratory alignment are within $1 \mathrm{~mm}$ and $0.14^{\circ}$, respectively. These results guarantee that any relative pointing offset and efficiency degradation are not severe. The fact that the beam offsets on the sky must be less than 2 arcseconds was critical during the final installation of the receiver optics on the telescope.

\subsection{Low Pass Filters}

In order to make simultaneous observations in four bands possible, the three LPFs should act as frequency dividers that route the signal from the subreflector to the appropriate receiver. These filters reflect signals in their stop-band and transmit signals in their pass-band, in a manner similar to normal microwave filters. For optimal performance, the beam incidence angles to the filters need to be restricted to less than $20^{\circ}$; the incidence angles in our design are $17^{\circ}$ and $15^{\circ}$ [11]. About $7 \%$ loss in reflection and transmission at each filter was predicted while 
actual measurements showed slightly higher degradation possibly due to other misalignments in the optics. The effective reflection surfaces of LPF1 and LPF3 are located on the outer surface of each filter. On the other hand the LPF2 is covered with a $1.2 \mathrm{~mm}$-thick Teflon layer beneath which reflection was found to occur.

\subsection{Aperture efficiency}

We estimated the aperture efficiencies at the four frequency bands from the cross scan observations of the planets using Equations (1) to (4) of [12]. In order to estimate receiver gains, we used hot and cold conditions, i.e. a microwave absorber in ambient temperature and an absorber immersed in liquid nitrogen. Attenuation in the atmosphere at each frequency band is corrected by measuring optical depth using the conventional sky dipping method. Table 1 summarizes the estimated aperture efficiencies with the corresponding measurement conditions.

Table 1. Aperture efficiencies

\begin{tabular}{|c|c|c|c|c|c|c|}
\hline $\begin{array}{c}\text { Frequency } \\
(\mathrm{GHz})\end{array}$ & Obs. Date & $\begin{array}{l}\text { Source } \\
\text { Name }\end{array}$ & $\begin{array}{c}\text { Elevation } \\
\text { (deg) }\end{array}$ & $\begin{array}{l}\text { Source Size } \\
(\operatorname{arcsec})\end{array}$ & $\begin{array}{c}\text { Brightness } \\
\text { Temperature }(\mathrm{K})\end{array}$ & $\begin{array}{c}\text { Aperture } \\
\text { Efficiency }(\%)\end{array}$ \\
\hline 22.235 & Oct 25,2012 & Jupiter & $30-60$ & 46.1 & $134 \pm 4$ & $65 \pm 1$ \\
\hline 43.122 & Oct 25,2012 & Jupiter & $30-60$ & 46.1 & $150 \pm 15$ & $62 \pm 2$ \\
\hline 86.243 & Oct 25,2012 & Venus & $30-60$ & 13.7 & $357.5 \pm 13[15]$ & $57 \pm 2$ \\
\hline 129.363 & Oct 25,2012 & Venus & $30-60$ & 13.7 & [16] & $38 \pm 3$ \\
\hline
\end{tabular}

Note : Aperture efficiency errors are $1 \sigma$, not including systematic errors arising from uncertainties in the brightness temperatures.

We have carried out simultaneous multi-frequency observations to test the four-band system with $\mathrm{H}_{2} \mathrm{O}$ and $\mathrm{SiO}$ maser lines. We chose the Orion $\mathrm{KL}$ that is one of the famous star forming regions. Figure 2 shows the results of simultaneous four-band observations.

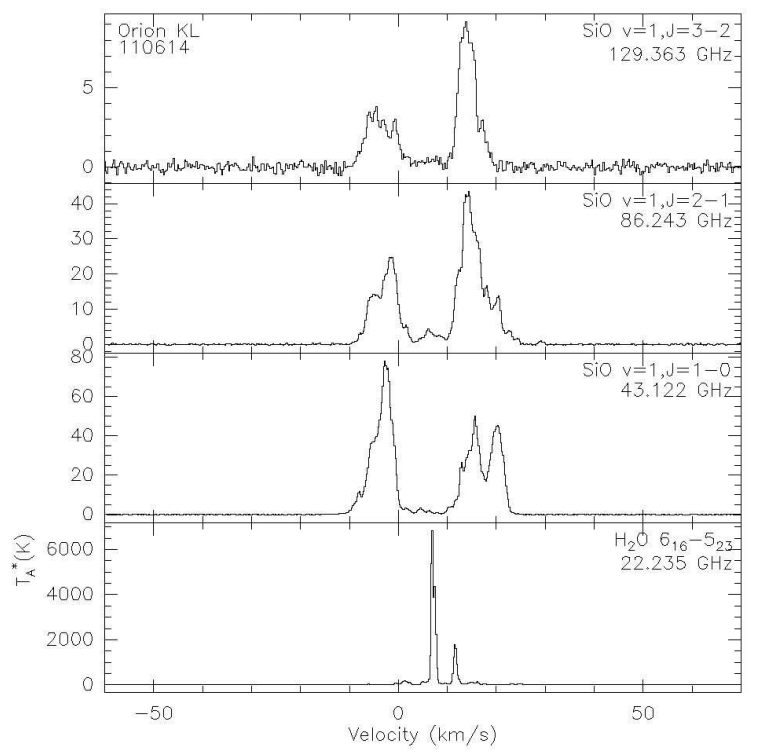

Figure 2. Simultaneous four-band observations of strong maser lines toward Orion-KL. 


\section{Summary}

We have developed a new millimeter wave receiver system that can perform simultaneous observations in up to four well-separated frequency ranges including 22, 43, 86 and $129 \mathrm{GHz}$ bands to calibrate tropospheric phase fluctuations for millimeter wave VLBI observations. Alignment of the four-band beams on the sky shows peak offsets within 2 arcseconds, a level that is less than $10 \%$ of the expected beam width at the highest-frequency band $(129 \mathrm{GHz})$. Telescope aperture efficiencies including receiver optics are $65 \%$ at $22 \mathrm{GHz}, 62 \%$ at $43 \mathrm{GHz}$, $57 \%$ at $86 \mathrm{GHz}$ and $38 \%$ at $129 \mathrm{GHz}$.

\section{References}

[1] A. J. Beasley \& J. E. Conway, 1995, in ASP Conf. Ser., 82, 327

[2] M. C. H. Wright, 1996, PASP, 108, 520

[3] E. Middelberg et al., 2005, A\&A, 433, 897

[4] Y. Asaki et al., 1996, Radio Sci., 31, 1615

[5] Y. Asaki et al., 1998, Radio Sci., 33, 1297

[6] M.-J. Rioja et al., 2002, in Proceedings of $6^{\text {th }}$ European VLBI Network Symposium, 57

[7] R. W. Porcas et al., 2003, in ASP Conf. Ser., 306, 39

[8] M. Honma et al., 2003, PASJ, 55, 157

[9] T. Jung et al., 2011, PASJ, 63, 375

[10]T. Sasao, 2003, in ASP Conf. Ser., 306, 53

[11]S. -T. Han et al., 2008, Int. J. Infrared Mill. Waves, 29, 69

[12]S. S. Lee et al., 2011, PASP, 123, 1398

[13]L. Page et al., 2003, ApJS, 148, 39

[14]A. Greve et al., 1994, A\&A, 286, 654

[15]B. L. Ulich et al., 1980, IEEE Trans. on Antennas and Propagation, AP-28, 367

[16]A. K. Fahd, 1992, NASA Technical Report No. 1992-1 